\title{
Cortical Cataract Grade 3
}

National Cancer Institute

\section{Source}

National Cancer Institute. Cortical Cataract Grade 3. NCI Thesaurus. Code C135199.

A stage of cortical cataract characterized by $50-90 \%$ of the intrapupillary space obscured by opacity. (Modified LOCS II) 